\title{
Use of recombinant human antithrombin concentrate in pregnancy
}

\author{
This article was published in the following Dove Press journal: \\ International Journal of Women's Health \\ 13 September 2013 \\ Number of times this article has been viewed
}

\author{
Lisa M Baumann Kreuziger ${ }^{1}$ \\ Tracy L Prosen ${ }^{2}$ \\ Mark T Reding' \\ 'Division of Hematology, \\ Oncology, and Transplantation, \\ University of Minnesota, MN, \\ USA; ${ }^{2}$ Obstetrics and Gynecology, \\ Maternal Fetal Medicine Center, \\ University of Minnesota, MN, USA
}

Correspondence: Lisa M Baumann Kreuziger

University of Minnesota, Mayo

Mail Code 480, 420 Delaware St SE,

Minneapolis, MN 55455, USA

$\mathrm{Tel}+\mathrm{I} 6126240123$

Fax +I 6126256919

Email bauma260@umn.edu

\section{Dear editor}

We read with great interest James et al's article "Prevention and treatment of venous thromboembolism in pregnancy in patients with hereditary antithrombin deficiency." The authors reported a case series of six women with antithrombin (AT) deficiency treated with plasma derived antithrombin concentrate (pdAT; Thrombate III ${ }^{\circledR}$, Grifols Therapeutics, Clayton, NC, USA). ${ }^{1}$ In contrast to these cases, we have managed three AT-deficient women over the past two years with low-molecular-weight-heparin during pregnancy and recombinant human antithrombin concentrate (rhAT; ATryn ${ }^{\circledR}$, GTC Biotherapeutics, Framingham, MA, USA) at delivery. In the Phase III trial leading to approval of rhAT, pregnant women required frequent dose modifications, and an alternate dosing regimen is currently recommended by the manufacturer. ${ }^{2,3}$ The detailed information provided in these cases may assist future providers in managing pregnant patients with AT deficiency.

\section{Case I}

Patient 1 is a 31-year-old female with a history of unprovoked pulmonary embolism at age 20 . Thrombophilia testing was completed due to her young age and a family history of pulmonary embolism in her brother. The patient was found to be AT deficient with an AT activity of $37 \%$. She was maintained on long term anticoagulation with warfarin. Her other past medical history was significant for supraventricular tachycardia, rheumatoid arthritis, endometriosis, pyelonephritis, Crohn's disease, and gastroesophageal reflux disease. The patient became pregnant in 2010 and was switched to enoxaparin at 4 weeks gestation. Her pregnancy was complicated by gestational diabetes, which was controlled by diet and exercise. Additionally, she had one admission due to supraventricular tachycardia and was found to have irregular uterine contractions. Due to the AT deficiency, anticoagulation was monitored using anti-Xa levels with goal peak level $>0.6 \mathrm{IU} / \mathrm{mL}$. At the highest dose, she required enoxaparin 120 units $(\sim 1.4$ units $/ \mathrm{kg}$ ) subcutaneously every 8 hours (Figure 1).

Patient 1 was admitted at 35 weeks and 4 days due to concerns for preterm labor. She was continued on enoxaparin until the evening of hospital day (HD) 2. She started rhAT on HD3 with a loading dose of $48.5 \mathrm{IU} / \mathrm{kg}$ (calculated from [100-baseline activity]/1.3) and maintenance dose of $11 \mathrm{IU} / \mathrm{kg} /$ hour (calculated from [100-baseline activity]/5.4). She underwent induction of labor via cervical ripening catheter and oxytocin when the AT level was in the normal range. Amniotomy was performed on 


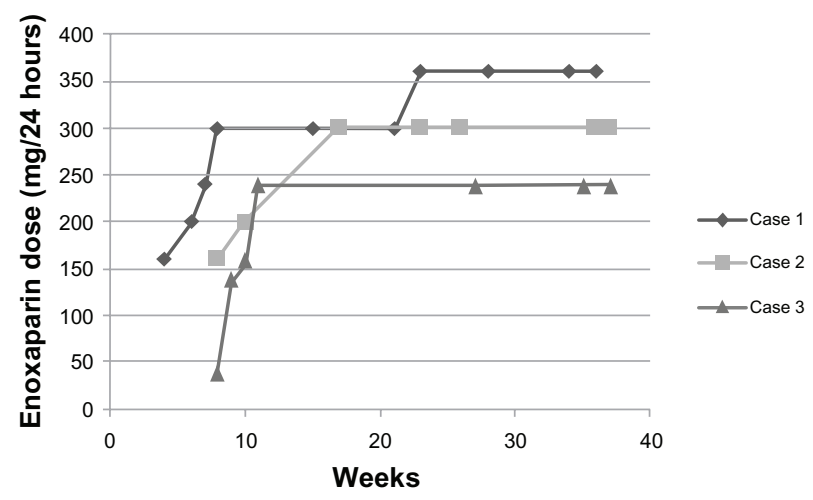

Figure I Daily dose of enoxaparin required to maintain therapeutic anti-Xa levels.

HD5, and the patient vaginally delivered a healthy infant weighing 2,268 grams on HD6. Apgar scores were 7 and 9 at 1 and 5 minutes, respectively. The estimated blood loss was $250 \mathrm{~mL}$. The rhAT infusion did not require dose modifications and the patient's AT levels remained between $90 \%-108 \%$ (Figure 2). Intravenous unfractionated heparin was started immediately postpartum and was adjusted by anti-Xa levels. Additionally, the patient was started on warfarin on the evening of delivery. Admission hemoglobin of the patient was $14.4 \mathrm{~g} / \mathrm{dL}$ and postpartum hemoglobin was $11.5 \mathrm{~g} / \mathrm{dL}$. She was continued on an unfractionated heparin and AT3 infusion until the international normalized ratio (INR) was greater than 2.0 and she was subsequently discharged.

\section{Case 2}

Patient 2 is a 40-year-old female with a past medical history significant for viral encephalitis and a left leg superficial thrombosis at age 32 while taking oral contraceptive pills. Her oral contraceptive pills were discontinued and she was treated with anti-inflammatory medication. At age 40, patient 2 was 8 weeks pregnant with her third child and sought evaluation for right calf and popliteal pain. She was diagnosed with a

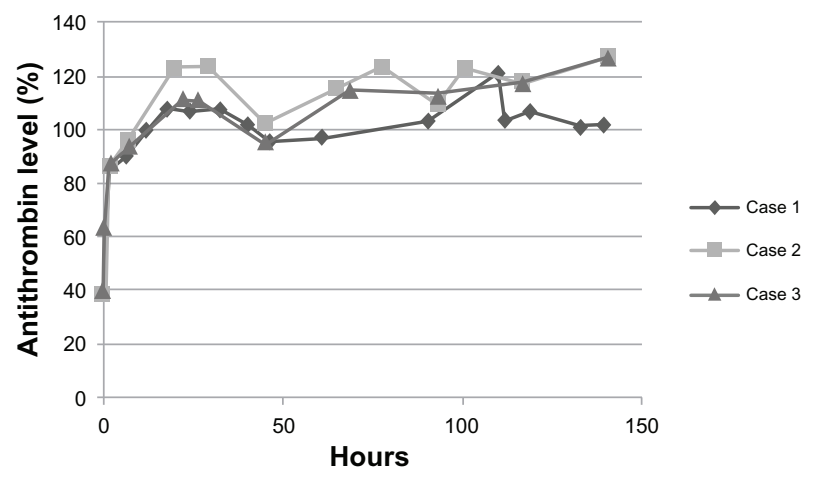

Figure 2 Antithrombin levels during treatment with recombinant human antithrombin concentrate. Normal antithrombin levels between $80 \%-125 \%$ at our institution. non-occlusive deep vein thrombosis of the distal femoral, popliteal, and posterior tibial vein. A hypercoagulable evaluation showed an antithrombin activity of $38 \%-41 \%$. Her sister, father, and daughter were subsequently diagnosed with antithrombin deficiency but have not experienced thrombosis. Patient 2 was initially treated with enoxaparin at $1.5 \mathrm{mg} / \mathrm{kg}$ daily, and was switched to twice daily dosing after the antithrombin deficiency was diagnosed (Figure 1). At the highest dosage, she required enoxaparin at $1.8 \mathrm{mg} / \mathrm{kg}$ twice daily to maintain therapeutic anti-Xa levels. Her pregnancy was otherwise uncomplicated and she was admitted at 37 weeks 4 days gestation for induction of labor. She received a bolus of $41.4 \mathrm{IU} / \mathrm{kg}$ and maintenance infusion of $10 \mathrm{IU} / \mathrm{kg}$ (calculated as above). Labor induction began once antithrombin levels had normalized. The patient vaginally delivered a healthy male with Apgar scores of 8 and 9 at 1 and 5 minutes, respectively. Estimated blood loss was $300 \mathrm{~mL}$. Her antithrombin levels remained between $96 \%-127 \%$ without adjustment of her infusion (Figure 2). An unfractionated heparin infusion was started 4 hours after delivery. She was discharged on HD9 on warfarin and twice daily enoxaparin until her INR was $>2.0$ two days later. She has been maintained on therapeutic anticoagulation due to her history of thrombosis in the setting of antithrombin deficiency.

\section{Case 3}

Patient 3 is a 27 -year-old female with a history of pulmonary embolism 4 days after cesarean delivery of her first child. She was treated with weight-based enoxaparin without monitoring of anti-Xa levels. The patient sought hematology evaluation when she was 8 weeks pregnant with her second child. A hypercoagulable work-up was notable for an antithrombin level of $63 \%$. Repeat testing showed antithrombin activity levels of $64 \%-73 \%$ with normal antigen levels. The patient had no family history of thrombosis. Due to the discrepancy between the amount of clinical heparin resistance and her antithrombin activity, sequencing of the SERPINC1 gene was completed. She was found to have a heterozygous mutation in the heparin binding site (c.218C > T, p.Pro73Leu) which is associated with type II antithrombin deficiency. To achieve therapeutic anticoagulation, the patient was treated with $1.8 \mathrm{mg} / \mathrm{kg}$ of enoxaparin. She did not require further increase in her enoxaparin dose after therapeutic levels were obtained (Figure 1). She underwent elective induction at 37 weeks 3 days after rhAT infusion was initiated. Her last dose of enoxaparin was 24 hours prior to induction. Patient 3 required a cesarean delivery on HD2 due to intrapartum spontaneous conversion to breech presentation. A healthy male infant was 
delivered with an estimated blood loss of 1,200 $\mathrm{mL}$ due to uterine atony. Eight hours postpartum the patient was started on heparin infusion and warfarin. She achieved therapeutic anticoagulation within 4 hours. Her AT activity remained in the normal range without adjustments of her rhAT infusion (Figure 2). On HD3, the patient developed right arm pain and was noted to have an occlusive superficial thrombus of the right basilic vein and a nonocclusive deep thrombosis of the distal right axillary vein associated with the peripherally inserted central catheter (PICC). The PICC was removed as it was considered the etiology of the thrombosis, and the patient continued the rhAT via a peripheral intravenous infusion. The patient's right arm pain resolved and she was discharged on HD8 after therapeutic anticoagulation with warfarin was achieved.

\section{Discussion}

In contrast to the Phase III trial of rhAT at delivery, the presented three patients did not require dose modifications to maintain therapeutic levels. ${ }^{4}$ The continuous infusion also avoids peak and trough levels that occur with intermittent dosing of the pdAT concentrate. ${ }^{1}$ Use of a recombinant product also removes the risk of blood-borne pathogen transmission, although no reported infectious transmissions with the pdAT have occurred. ${ }^{1}$ The rhAT is more expensive than pdAT and carries a theoretic risk of immunogenicity. Our patients' antibody testing per the US Food and Drug Administration's post-marketing surveillance is pending, and no neutralizing antibodies were detected in other reported cases. ${ }^{2}$ One of our patients experienced a catheter-associated thrombosis, and recurrent thrombosis has also been reported in patients treated with plasma-derived AT concentrates. ${ }^{5-7}$ Even when AT replacement is given, physicians should remain vigilant about thrombosis and avoid other provoking factors if possible. Overall, the availability of two products to treat women with AT deficiency at delivery allows providers to select the management strategy best suited for their patient and institution.

\section{Acknowledgments}

We would like to thank Kerry Hansen, RN, for assistance with data abstraction.

\section{Disclosure}

Lisa M Baumann Kreuziger and Tracy L Prosen have no interests which might be perceived as posing a conflict or bias. Mark T Reding began serving as an advisory board member for GTC Biotherapeutics after the above cases were managed. Funding for Lisa Baumann Kreuziger's time to complete this work was supported by a NIH T32 training grant (5T32HL00706).

\section{References}

1. James AH, Konkle BA, Bauer KA. Prevention and treatment of venous thromboembolism in pregnancy in patients with hereditary antithrombin deficiency. Int J Womens Health. 2013;5:233-241.

2. Tiede A, Tait RC, Shaffer DW, et al. Antithrombin alfa in hereditary antithrombin deficient patients: a phase 3 study of prophylactic intravenous administration in high risk situations. Thromb Haemost. 2008;99(3): 616-622.

3. GTC Biotherapeutics, Inc. Antithrombin (Recombinant) ATryn for Injection: Highlights of Prescribing Information. Framingham, MA: GTC Biotherapeutics, Inc; 2010. Available from: http://atryn.com/pdf/ ATryn_PI_0409.pdf. Accessed July 31, 2013.

4. Tanimura K, Ebina Y, Sonoyama A, Morita H, Miyata S, Yamada H. Argatroban therapy for heparin-induced thrombocytopenia during pregnancy in a woman with hereditary antithrombin deficiency. $J$ Obstet Gynaecol Res. 2012;38(4):749-752.

5. Hidaka N, Hachisuga M, Tsukimori K, Onohara T, Maehara Y, Wake N. Intrapartum placement of an inferior vena cava filter for a woman with hereditary antithrombin III deficiency: its role in the prevention of fatal pulmonary embolism. J Obstet Gynaecol Res. 2008;34(1): 95-99.

6. Yamada T, Yamada H, Morikawa M, et al. Management of pregnancy with congenital antithrombin III deficiency: two case reports and a review of the literature. J Obstet Gynaecol Res. 2001;27(4):189-197.

7. Hellgren M, Tengborn L, Abildgaard U. Pregnancy in women with congenital antithrombin III deficiency: experience of treatment with heparin and antithrombin. Gynecol Obstet Invest. 1982;14(2):127-141. 


\section{Authors' reply}

\section{Andra H James' \\ Barbara A Konkle ${ }^{2,3}$ \\ Kenneth A Bauer ${ }^{4}$}

'Department of Obstetrics and Gynecology, University of Virginia, Charlottesville, VA, '2Puget Sound Blood Center, Seattle, WA

${ }^{3}$ Department of Medicine, University of Washington, Seattle, WA, ${ }^{4}$ Department of Medicine, Beth Israel Deaconess Medical Center and VA Boston Healthcare System, Harvard Medical School,

Boston, MA, USA

\section{Correspondence: Andra $\mathrm{H}$ James}

University of Virginia, Department of Obstetrics and Gynecology,

PO Box 8007/2, Charlottesville, VA, USA

Tel + I 434 98। 1904

Email andra.james@virignia.edu

\section{Dear editor}

We would like to thank the authors for sharing their experience with the use of recombinant antithrombin concentrate in pregnancy and for adding to the body of literature on the management of this rare condition.

\section{Disclosure}

The authors report no conflicts of interest in this communication.

\section{Publish your work in this journal}

The International Journal of Women's Health is an international, peerreviewed open-access journal publishing original research, reports, editorials, reviews and commentaries on all aspects of women's healthcare including gynecology, obstetrics, and breast cancer. The manuscript management system is completely online and includes

a very quick and fair peer-review system, which is all easy to use. Visit http://www.dovepress.com/testimonials.php to read real quotes from published authors. 